\title{
Role of cardiac MRI and CT in Fontan circulation
}

\author{
Michael Yeong ${ }^{1 *} \mathbb{D}$, Will Loughborough ${ }^{2}$, Mark Hamilton ${ }^{3}$ and Nathan Manghat ${ }^{3}$
}

\begin{abstract}
A Fontan circulation is a series of palliative surgical procedures, which result in the diversion of the systemic venous return into pulmonary arterial circulation without passing through a ventricle. It is one of the available surgical strategies for patients with cardiac defects that preclude a successful bi-ventricular repair, which encompass a range of complex anatomy. This surgical repair has gone through a series of modifications since the concept was introduced in 1971. Echocardiography remains a vital tool in assessing patients with Fontan circulations but its limitations are well recognised. Cross-sectional imaging modalities such as cardiac MRI and CT are essential components in the systematic clinical evaluation of these patients. The purpose of this review is to understand the information that can be obtained with each cross-sectional modality as well as highlight the challenges that each modality faces.
\end{abstract}

Keywords: Fontan, Cardiac MRI, Cardiac CT, Cross-sectional Imaging

\section{Background}

A Fontan circulation is a series of palliative surgical procedures, which result in the diversion of the systemic venous return into the pulmonary arterial circulation without passing through a ventricle. It is one of the available surgical strategies for patients with cardiac defects that preclude a successful repair with a biventricular circulation. These defects may include hypoplastic left heart syndrome, tricuspid atresia, mitral atresia, double-inlet left ventricle, and unbalanced atrioventricular septal defect.

In 1971, Francois Fontan and Eugene Baudet proposed a palliative procedure for tricuspid atresia [1]. This comprised of (1) anastomosis of the superior vena cava (SVC) to the distal right pulmonary artery with ligation of the SVC below the anastomosis, (2) division of the proximal right pulmonary artery from the main pulmonary artery, (3) anastomosis of the right atrium to the distal left pulmonary artery with an aortic homograft, (4) closure of the atrial septal defect and (5) ligation of the main pulmonary artery. Since then, there have been different modifications to this procedure, collectively categorised as 'atriopulmonary Fontan'. This method was the

\footnotetext{
* Correspondence: Michael.yeong@gmail.com

${ }^{1}$ Department of Paediatric Cardiology, Bristol Royal Hospital for Children, University Hospitals Bristol NHS Foundation Trust, Upper Maudlin St, Bristol BS2 8HW, UK

Full list of author information is available at the end of the article
}

predominant technique until the 1980's when the late complications such as sinus node dysfunction, thrombosis and right-sided pulmonary venous obstruction became increasingly apparent. Whilst this method is no longer employed, adult patients with this style of Fontan circuit are regularly encountered in clinical practice.

The initial concept of the atriopulmonary Fontan was based on the thought that the pulsatile atrium could act as a pump and improve pulmonary blood flow [2]. However, over time, the right atrium dilates and turbulent flow developed within the chamber resulting in energy loss (Fig. 1). Dynamic experiments performed in 1988 demonstrated the benefits of reduction of turbulent flow to the pulmonary arteries which led to the development of the lateral tunnel total cavopulmonary connection and more recently, the extra-cardiac conduit using prosthetic polytetrafluoroethylene conduit external to the atrium (Fig. 1) [3, 4].

A different variation of the Fontan is the Kawashima procedure. This technique has been employed for palliation of 'single ventricle' hearts in the presence of an interrupted inferior vena cava (IVC) with azygous or hemiazygous continuation (Fig. 2). The procedure comprise of division of the SVC distal to the azygous drainage and anastomosis to the branch pulmonary arteries resulting in near total drainage of systemic venous return to the pulmonary arterial circulation, apart from the hepatic and coronary circulation which drains into 


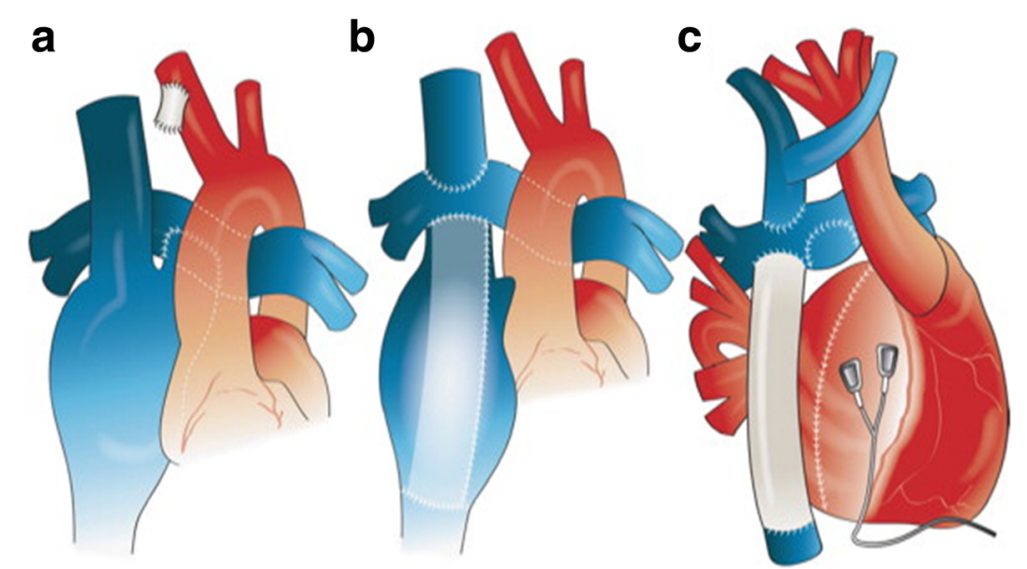

Fig. 1 Different types of Fontan. Graphical illustration of the different Fontan surgeries performed in the last 5 decades: (a) atrio-pulmonary Fontan with the dilated right atrium to the branch pulmonary arteries (left); (b) lateral tunnel Fontan with anastomosis of the SVC to the superior aspect of the right pulmonary artery (RPA), right atrium to the inferior aspect of the RPA and placement of baffle within the right atrium directing IVC flow into RPA (centre); and (c) extra-cardiac conduit with a Gore-Tex conduit directing IVC flow to the RPA (right). Re-produced from Mondesert et al. [69] with permission from Elseiver

the heart directly (Fig. 2) [5]. The distinctive risk of developing pulmonary arteriovenous malformations (PAVM) post Kawashima has led to the re-direction of the hepatic veins to the pulmonary arteries thus completing a Fontan and subsequent reduction in PAVM [6].

Echocardiography is the most widely used imaging modality in assessing patients with congenital heart disease. Whilst echocardiography remains a vital imaging assessment modality as patients grow into adulthood, there is an appreciation that the quality of echocardiographic images may be degraded due to diminishing acoustic windows. Multiple factors could be implicated such as higher BMIs, chest wall deformity and particularly in patients who have had prior surgical procedures [7].
Cardiac MRI (CMR) is utilised regularly in addition to echocardiography for long-term monitoring of Fontan patients [8]. It is very appealing as it avoids ionising radiation, though it does involve longer acquisition time but is able to provide a wealth of anatomical and physiological information. Cardiothoracic CT remains a valuable adjunct in situations where patients have contraindications to CMR or in specific clinical situations as described later.

\section{Anatomy}

Compared to echocardiography, a major advantage of CMR is the ability to assess the cardiac anatomy in multiple planes with high spatial resolution. With a combination of dark-blood or cinematic bright-blood imaging, it is possible to obtain excellent morphologic and

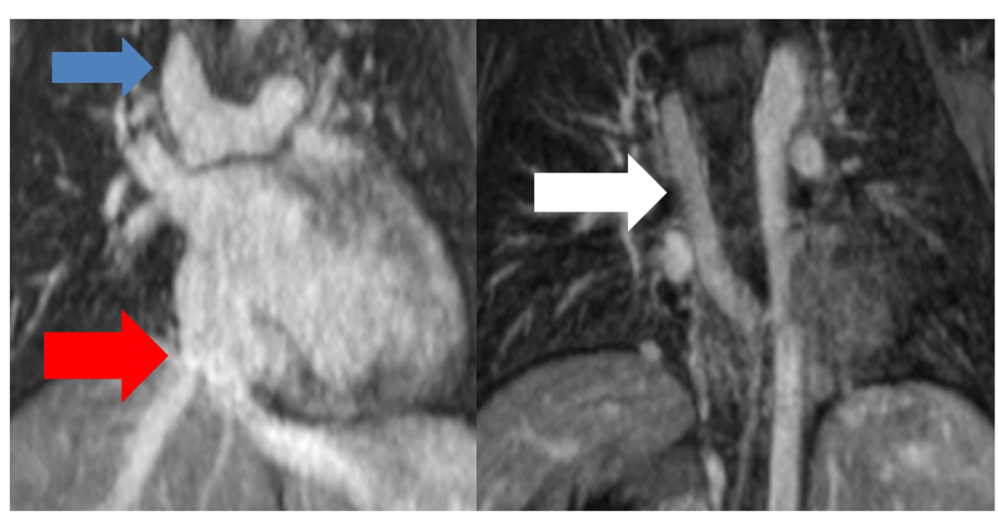

Fig. 2 Kawashima procedure. Figure on the right demonstrating an interrupted infra-hepatic inferior vena cava and it is continuous with the dilated azygous venous system (white arrow), which drains into the right superior vena cava (blue arrow). The superior vena cava has been anastomosed to the branch pulmonary arteries. Figure on the left demonstrating the hepatic veins drain directly into the right atrium (red arrow). Thus majority of the systemic venous blood is directed into the branch pulmonary arteries apart from the hepatic and coronary venous blood 
functional images of the heart [9-11]. This is vital given the diverse single ventricle anatomy in Fontan circulations.

The excellent blood-tissue contrast in balanced SSFP (steady state free precession) cine bright-blood imaging used on a contiguous stack of axial, coronal and sagittal images enables the visualisation of the Fontan pathway and the morphology of the intra-cardiac structures [12]. In addition, smaller structures such as coronary arteries have been shown to be reliably detected by MR angiography [13] or using 3D volumetric datasets [14].

Anatomical complications in the Fontan circuit can be assessed using CMR. This is important as the circuit relies on low pressure and minimal obstruction to flow. The pulmonary veins may be compressed by the dilated right atrium in the atriopulmonary Fontan [15]. It is also important to evaluate for stenosis in the caval veins and pulmonary artery anastomoses [16]. Compression of the central portion of the pulmonary arteries may be more common in Fontan patients with a reconstructed aorta (Damus-KayeStansel anastomosis). Occasionally this may be stented to maintain patency, which makes it difficult to assess using cine SSFP due to its sensitivity to magnetic field inhomogeneity. Different sequences such as spoiled gradient echo cine or black-blood turbo-spin echo imaging may be able to provide some information but does not exclude in-stent stenosis.

Gadolinium-enhanced MR angiography (MRA) can also be performed to highlight the vascular anatomy and this method has been shown to be accurate to diagnose abnormalities of the systemic and pulmonary veins, pulmonary arteries and aorta (Fig. 3) [17]. Images can be acquired over multiple phases thus highlighting different parts of the vascular anatomy over time. With the availability of new intra-vascular gadolinium-based contrast, newer sequences have been used to image the Fontan circuit in steady state [18].

\section{Ventricular volumetric and function}

CMR is the preferred method for the quantification of ventricular volume and systolic function in both normal [19] and abnormal hearts [20]. Importantly, CMR has been shown to be accurate in the quantification of ventricular volumes in univentricular hearts and more reproducible than echocardiography [21].

Ventricular volumes can be measured from a contiguous axial or short-axis stack of the single ventricle, using Simpson's method of discs. There is no clear consensus on which imaging plane is optimal for ventricular volumetric assessment and the merits of each plane has been described [22, 23].

It has been suggested that the systemic ventricle in Fontan patients often have an indexed end-diastolic volume [24] similar to normal ventricles however, the "normal" reference range for a univentricular heart remains elusive. Serial assessment of the ventricular volume is important as a dilated ventricle (end-diastolic volume greater than $125 \mathrm{ml} /$ body surface area $^{1.3}$ ) is associated with poorer outcomes [25], therefore a standard analysis technique is vital. In addition, it may be worthwhile evaluating the size and function of the secondary

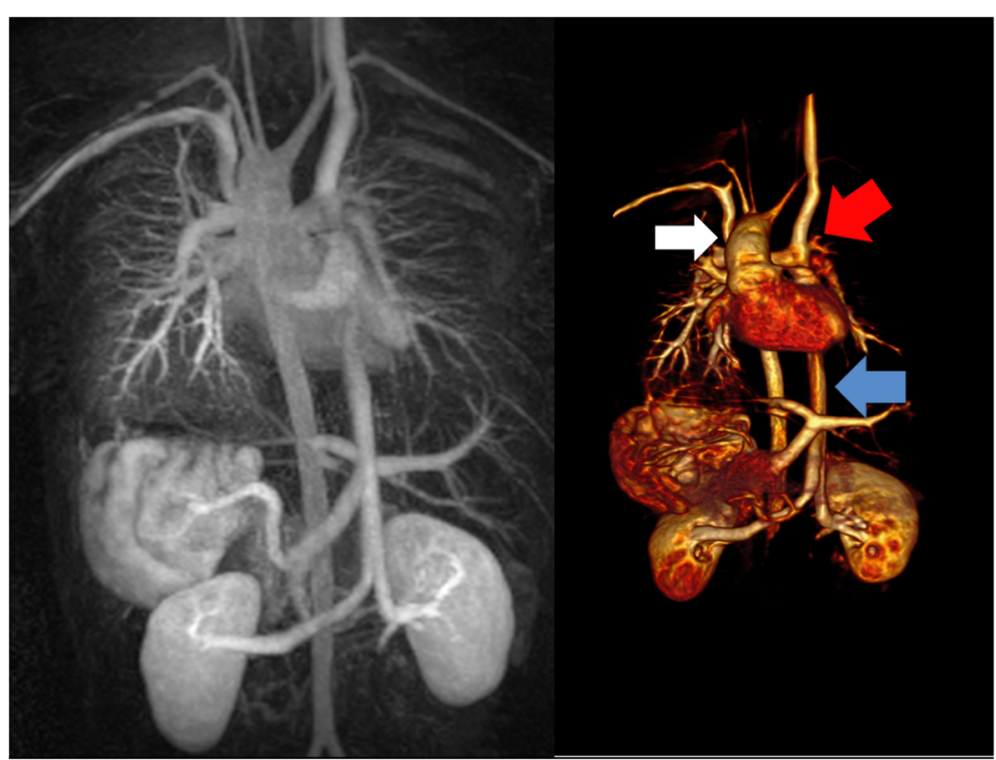

Fig. 3 Gadolinium-enhanced angiography. Situs inversus, with atrio-ventricular discordance, pulmonary atresia, right aortic arch and bilateral SVC. Repaired with bilateral SVC to branch pulmonary anastomosis and with extra-cardiac Fontan. Time resolved gadolinium MRA performed to evaluate the vascular connections. Angiography dataset can be rendered using different techniques: Maximal Intensity Projection (MIP) on the left and 3D volume on the right 
ventricle as it has been suggested that it may augment the work performed by the primary ventricle resulting in improved exercise capacity [26].

The systemic ventricle in the Fontan circuit has been demonstrated to have a reduced ejection fraction [24] though the reasons for it is not completely well understood. It might be that the (often) systemic right ventricles are not ideally suited to the systemic circulation and become hypokinetic in radial and longitudinal function. It has also been suggested that the hypertrophied ventricle had decreased compliance thus filling, resulting in reduced end-diastolic volume with normal endsystolic volume, and effectively reducing the ejection fraction [24]. Measurement of ejection fraction is made by employing Simpson's method and when done on a short-axis stack, it provides an evaluation of radial function of the single ventricle. What is perhaps not as well appreciated or quantified is the longitudinal shortening of the systemic ventricle. At the present, there is no data on assessment of longitudinal function using CMR in single ventricles in the literature and may be a new area of research in the future.

\section{Flows}

Historically flow measurement using invasive thermodilution techniques during cardiac catheterisation have been the gold standard. Non-invasive techniques based on echocardiography using spectral Doppler velocitytime integral and vessel area have been developed but faced with considerable limitations in terms of accuracy and reproducibility [27]. CMR flow assessment has been validated extensively in vivo and in vitro [28] with less than $5 \%$ difference when compared to Fick calculation and thermodilution. Using phase-contrast cine MRI (also referred to as phase-velocity or velocity-encoded cine), CMR can accurately measure flow in vascular structures, relying on the fundamental concept that nuclei flowing through a specifically designed magnetic field gradient accumulates a predictable phase shift that is proportional to the flow velocity. Systolic and diastolic blood flow is determined by measuring the velocity of flow in each voxel of the vessel of interest at each time point during the cardiac cycle and multiplying by the vessel cross-sectional area. This allows calculation of indices such as peak and mean velocity, stroke volume, regurgitation and cardiac output.

In the Fontan circulation, phase contrast MRI can be used to assess the distribution of branch pulmonary artery flows to each lung thus providing a haemodynamic assessment of any significant obstruction. Evaluating split flows in the branch pulmonary arteries can be performed by estimation of the inflow (caval return) and assessment of the left pulmonary artery (LPA) flow. Difference between the inflow and LPA flow gives right pulmonary artery (RPA) flow. RPA flow is often impractical to directly measure due to the position of the caval anastomoses.

The flows in each individual pulmonary vein can also be measured and the difference between cumulative pulmonary vein flow and pulmonary arterial flow provide a quantification of aorto-pulmonary collateral burden [29]. An alternative technique to estimate the aorto-pulmonary collateral burden is the difference between the ascending aorta flow and total systemic venous flow [30]. Evaluating collateral burden may play a crucial role in Fontan as this shunt results in additional volume load to the single ventricle and may benefit from intervention such as coiling.

In addition, phase-contrast MRI can be used to quantify the magnitude of regurgitation by assessing the regurgitation fraction and volume in the atrio-ventricular and semi-lunar valves. It can also provide a flow-velocity map across a stenotic valve as well. Nevertheless, phasecontrast MRI has not replaced echocardiography in the assessment of valve stenosis and regurgitation; rather it provides supplementary information.

Conventional flow measurements are performed in 2D. Advancement in 3D spatial encoding has provided the opportunity to measure flow and motion patterns in a 3D volume, which is known as 3D velocity encoding. Thus ECG synchronised 3D flow mapping using $3 \mathrm{D}$ velocity encoding (also known as 4D flow) can be utilised to visualise global and local blood flow characteristics in any vascular target of choice [31]. Application of 4D flow in a cohort of various Fontan circuit connections revealed different energy loss with different connections (Fig. 4) [32]. Increasing power loss is found to be associated with reduction in aerobic exercise capacity [33]. Application of this may lead to individualised modification of the Fontan circuit for optimal flow dynamics and hence exercise capacity [34].

\section{Myocardial fibrosis}

Myocardial fibrosis has been suspected to be a contributor to progressive deterioration in ventricular function in univentricular hearts. Focal areas of fibrosis can be evaluated using CMR technique called delayed enhancement. It requires an intravenous bolus of a paramagnetic agent (gadolinium) followed by image acquisition typically 5-20 min after contrast administration. Areas of scar appear bright on delayed enhancement imaging due to slower extra-cellular gadolinium contrast clearance kinetics in scar tissue. Recently, the presence and extent of delayed enhancement in the myocardium correlated with regional and global ventricular dysfunction as well as non-sustained ventricular tachycardia in these patients [35]. 


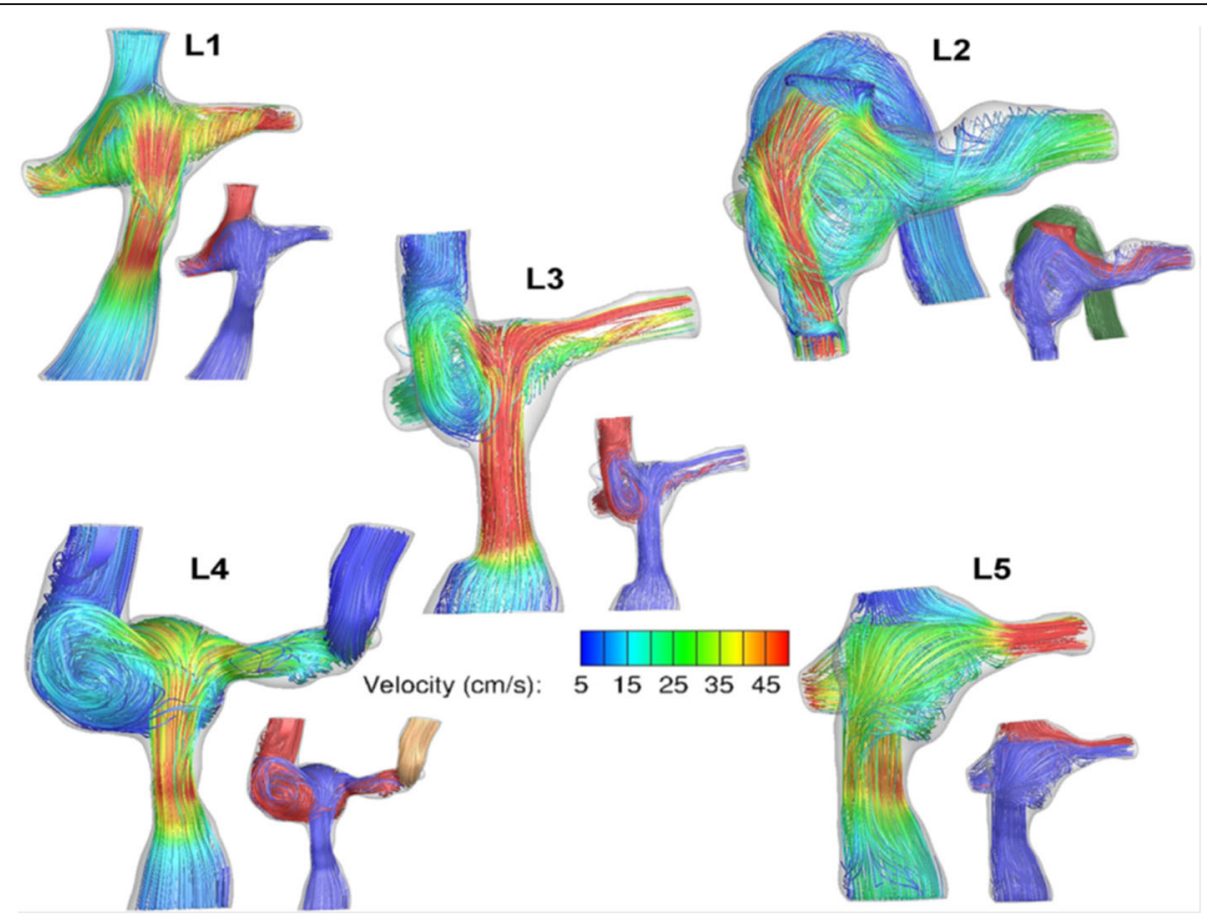

Fig. 4 4D Flow Dynamics. Computational flow dynamic demonstrating velocities streamline colour coded by local velocity magnitude within the different Fontan pathways. Re-produced from Haggerty et al. [34] with permission from Elseiver

In addition to focal fibrosis, CMR is currently utilised to evaluate for diffuse fibrosis. Diffuse fibrosis is viewed as the common pathological pathway toward deterioration in myocardial function in various pathologies [36, 37]. The two techniques commonly used in assessing diffuse fibrosis are myocardial T1 mapping and extracellular volume fraction (ECV) mapping [38]. T1 time is relatively specific for different tissues. Increased collagen (fibrosis), oedema (inflammation) and protein infiltration (amyloidosis) all prolongs the pre contrast or "native" T1 time whereas lipid, haemorrhage and siderosis all may reduce the T1 time. ECV is derived from the precontrast and post-contrast T1 in the myocardium and blood, combined with haematocrit. It reflects the volume fraction of heart tissue that is not taken by cells and it acts as a surrogate marker for fibrosis [39].

At present stage, the application of native $\mathrm{T} 1$ and ECV in congenital heart disease remains in the research domain and may prove useful clinically in the future as a biomarker for disease status.

\section{Thrombus}

Thromboembolic disease is recognised as an early and late complication after Fontan operation [40]. The aetiology is not completely defined but in part may be related to presence of foreign material, systemic venous stasis and low cardiac output, a common feature amongst all forms of Fontan operations [41]. Reports in the literature seem to indicate imbalance of coagulation factors after Fontan operations may play an additional role in the formation of thrombus [42]. Using brightblood cine imaging techniques, thrombus would appear as a low-signal intensity mass within Fontan pathways or ventricular chambers [43]. Newer and sensitive thrombus detection techniques have been employed; based on gadolinium contrast imaging with parameters adjusted to demonstrate the low-signal intensity avascular thrombus surrounded by higher-signal intensity contrast filled blood pool and myocardium [44]. As the incidence of thrombus in this population is significant (approximately 12\%) and carries a substantial burden of morbidity and mortality, emphasis has to be placed to prevent and detect the formation of clots [45].

\section{Challenges in CMR imaging}

The growing appreciation of the diagnostic value of CMR in congenital heart disease has paralleled its application in the clinical world. Nevertheless, the complexity and sensitivities involved in CMR image acquisition poses several technical challenges.

\section{Time}

Most CMR sequences require the patient to maintain breath-hold to minimise cardiac and respiratory motion. This is usually done in expiration (as diaphragmatic position is more stable than in inspiration) over about 
10 to $20 \mathrm{~s}$. The development of parallel imaging techniques have significantly reduced the acquisition time without a heavy penalty to spatial and temporal resolution [46]. Nevertheless, if a patient cannot perform good quality expiratory breath holds, inspiratory or free breathing techniques can be used, bearing in mind that different breathing technique used affects haemodynamics [47].

Free-breathing signal averaged cine imaging can be deployed as an alternative method whereby multiple acquisitions are "averaged" to reduce the respiratory motion effects however the duration of each sequence hence the study would undoubtedly be prolonged [48]. A newer technique that may prove useful in the future is the free breathing respiratory-triggered (navigator) cardiac gated cine imaging [49]. This technique is based on data acquisition at the end-expiratory phase of the respiratory cycle and quality of the images as similar to breath-hold images. This is particularly handy in patients who are not able to breath hold consistently such as young children.

\section{Rhythm disturbance}

The most commonly used method in CMR cine imaging uses retrospective ECG gating. High-quality images depending on a steady heart rhythm as irregularities result in blurry images. Most manufacturers include arrhythmia rejection algorithms that will reject data from R-R cycles that differ from a set parameter. This may substantially prolong the acquisition time and exceed the breath-hold capacity of the patient. An alternative strategy is to only acquire data between end diastole and mid diastole. This is called prospective ECG gating whereby the acquisition starts at the onset of the $\mathrm{R}$ wave. A variable amount of late-diastole is not evaluated, and the acquisition begins again at the next $R$ wave. This technique can greatly improve image quality but can appear jerky and lead to error in end diastolic volume assessment if there is dyssynchrony or the gating does optimally not start at the $\mathrm{R}$ wave. This method is usually adequate to evaluate systolic function. If the variation in the $\mathrm{R}-\mathrm{R}$ cycle is wide, such as in atrial fibrillation, real time cine imaging may be acquired with all the data acquired within 1 cardiac cycle at a cost of spatial and temporal resolution. As (in particular) end-systolic volume may be over-estimated with this method, this in turn would lead to an under-estimation of stroke volume and ejection fraction [50].

\section{Devices/implants}

Patients with Fontan circulations regularly undergo trans-catheter or surgical procedures that may involve implantation of devices that contain ferro-magnetic material. This may include endovascular coils or plugs for occlusion of collateral vessels, pulmonary artery stents for localised stenosis, closure devices to occlude fenestrations or baffle leaks, mechanical or bioprosthetic valves for dysfunctional valves, pacing wires with pacemaker (PPM) or implantable cardioverter-defibrillator (ICD) for rhythm disturbances. The magnitude of artefacts produced is proportional to the amount of ferromagnetic materials contained within these devices, which can have significant impact on the quality and interpretation of the study (Figs. 5 and 6) [51, 52] with SSFP cine imaging. Thus, implants made of platinum or Nitinol exudes fewer artefacts, but not zero. Some of the artefacts may be reduced by converting to spoiled gradient echo cine imaging for intra-cardiac evaluation or to a T1-weighted black-blood turbo spin echo imaging for vascular anatomy. Despite of this, some studies continue to be uninformative or non-diagnostic and alternatives need to be considered.

Recently published guidelines have indicated that MRI can be performed safely in patients with an implanted PPM or ICD (MRI conditional or not) as long as strict safety conditions are met [53]. This has been a major shift as previously these devices are generally considered a contra-indication to a MRI. However, significant artefact may be generated which prevents a diagnostic study being achieved, often because the pacing box is implanted in the left anterior chest wall.

Historically concerns have been raised regarding potential life-threatening interactions between PPM/ICD and MRI $[54,55]$. This has led to the development of more MRI conditional devices [56]. Upgrades in device programming combined with improved lead design has reduces the risk of complication. Needless to say, in these patients, MRI studies should be performed in a dedicated centre with appropriate experience, monitoring equipment (such as pulse oximeter) and supporting

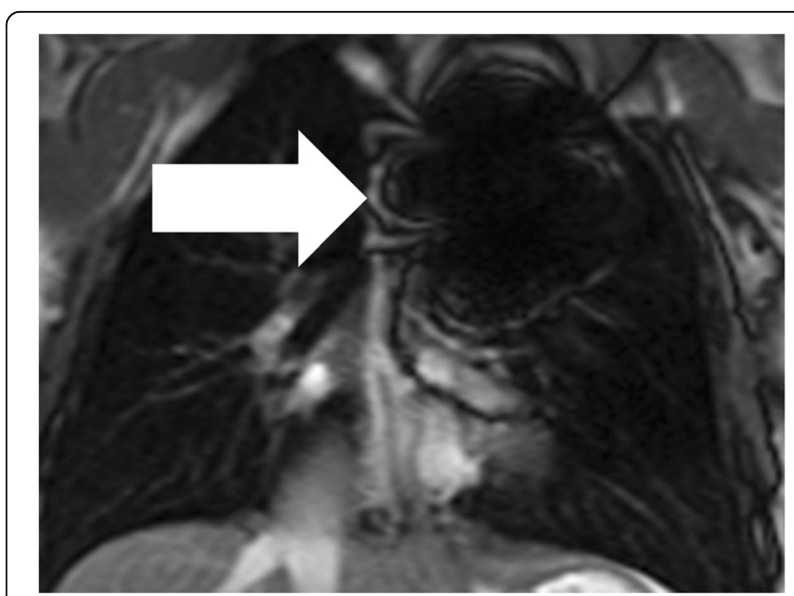

Fig. 5 Blooming artefact. Significant artefact (arrow) obscuring the left hemi-thorax due to stainless steel coils used to occlude a left modified Blalock-Taussig shunt, making the study non-diagnostic 


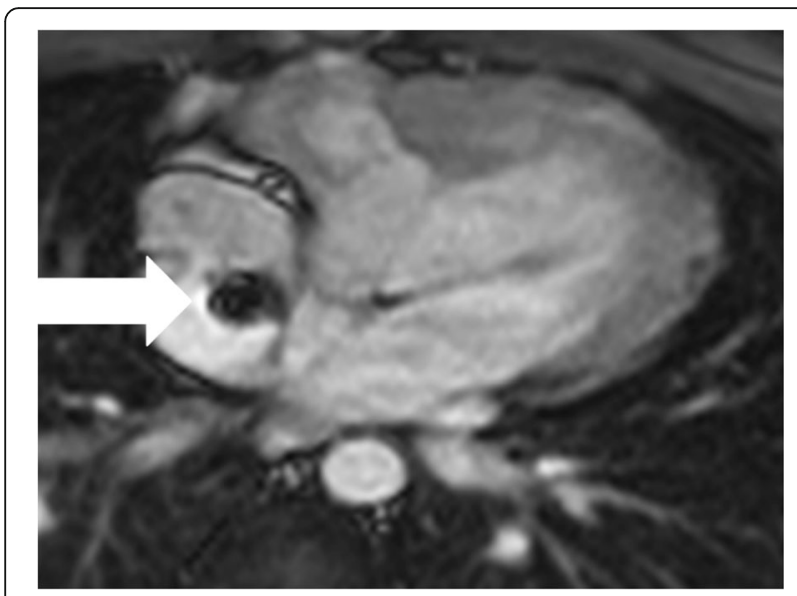

Fig. 6 Susceptibility artefact. Susceptibility artefact (arrow) from the fenestration closure device obscuring part of the Fontan pathway. The magnitude of the artefact is less due to lower ferro-magnetic properties of the closure device, which comprised of nickel and titanium

expertise. At present, performing cardiac MRI in the presence of abandoned pacemaker wires is still strongly discouraged due to the lack of published evidence and risk of inducing current induction respectively [57].

\section{Contrast toxicity/NSF}

Gadolinium-based MRI contrast agents have been used extensively to study vascular structures within the body. The gadolinium contrast agents available in the market are pre-dominantly excreted via the renal system [58]. The ionic form of gadolinium is toxic to the body thus is chelated with other molecules to increase its stability in physiological conditions allowing safe excretion from the body. There is a rare but potential fatal complication of using gadolinium-based agents called nephrogenic systemic fibrosis (NSF). The exact pathological process behind NSF is not fully understood but it is thought that renal dysfunction prolong the half-life of gadolinium chelates thus increasing the risk of dissociation of the gadolinium ion into the tissue. An inflammatory process is produced resulting in fibrosis of the affected tissues. Review of all reported cases NSF in the literature found that the majority of the patients were given more than $0.1 \mathrm{mmol} / \mathrm{kg}$ of a non-ionic gadolinium chelate and had severe renal insufficiency with $85 \%$ had glomerular filtration rate $($ GFR $)<15 \mathrm{ml} / \mathrm{min} / 1.73 \mathrm{~m}^{2}$ and another $6 \%$ had GFR $<30 \mathrm{ml} / \mathrm{min} / 1.73 \mathrm{~m}^{2}$ [59]. Thus it is prudent to assess the renal function if there are risk factors. If there is no good clinical indication, then the administration of a gadolinium chelate should be avoided if the GFR is less than $30 \mathrm{ml} /$ $\min / 1.73 \mathrm{~m}^{2}$.

More recently, there are reports in the literature about gadolinium deposition in the cerebral tissue in some patients who undergo four or more contrast MRI studies in the absence of renal or hepatic dysfunction $[60,61]$. This is subsequently confirmed on electron microscopy of autopsy tissue. It appears to be associated with administration of linear gadolinium agents and not seen with the macro-cyclic agents. At this moment, the clinical significance of this is unclear but nevertheless caution is required and risks and benefits of the use of gadolinium must be weighed carefully.

\section{Computed tomography in the Fontan circuit}

With the advent of the latest generation CT systems providing fast gantry rotation speeds and higher temporal resolution, whole heart volumetric acquisition, submillimetre isotropic spatial resolution, a few milliSievert radiation dose and state-of the-art semi-automated postprocessing software, most cardiac $\mathrm{CT}$ investigations can be performed and analysed with increasing ease. The use of Computed Tomography (CT) in congenital heart disease (CHD) has improved the accuracy of anatomical imaging and may allow the reduction of invasive catheter studies and thus the reduction in overall patient radiation burden [62]. CT can also facilitate a more focused approach to the catheter study.

However it is easy to perform a poor study without some specific considerations.

Whilst ventricular functional imaging is possible using $\mathrm{CT}$, this is at the expense of a higher radiation dose as more phases of the cardiac cycle must be optimally/adequately imaged, and standard software packages do not lend themselves easily to the single/complex ventricle. Flow assessment is not directly possible by CT and is left to $\mathrm{CMR}$ and echocardiographic assessment. Indirect statements of flow and physiology can sometimes be made from anatomical CT image interpretation such as by means of changes in contrast density and assessment of chamber or great vessel size and opacification.

\section{Fontan circuit considerations}

There are unique anatomical and physiological considerations when imaging Fontan circuits with CT. Typically contrast injected into the upper arm passes to the superior vena cava, then to the pulmonary arterial circuit, preferentially filling the RPA and thence the right sided veins. The systemic circulation is then enhanced and it is only after significant contrast has returned from the lower body via the inferior vena cava that the LPA is enhanced. Early phase CT (typical in a standard CT pulmonary angiogram) is thus likely to show excessive (and non diagnostic) contrast in the RPA and a poorly opacified (and non diagnostic) LPA. Additional slow-flowing unopacified blood flowing in from the inferior vena caval/extra cardiac conduit connection causes notable 

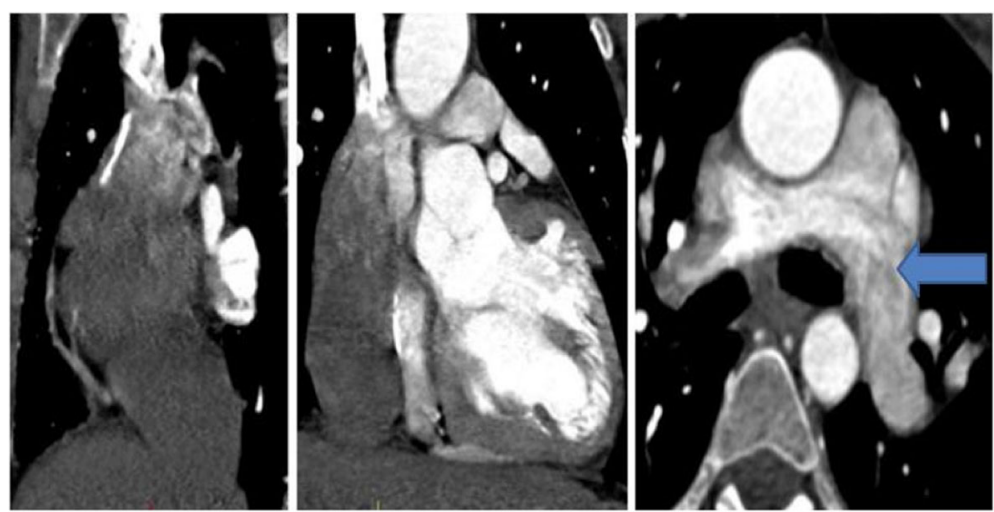

Fig. 7 Single contrast phase, single acquisition (as for standard CT pulmonary angiogram protocol). In the two images on the left, unopacified blood enters from the inferior Fontan connection causing mixing with contrast. This result in a pseudo-thrombus in the main pulmonary arteries - see blue arrow in far right image

mixing artefact, resulting in a pseudo thrombus or pseudo-pulmonary embolism (Fig. 7).

The details of variable RV/LV dominant morphology and function along with incompetent systemic/atrioventricular valves are important as these will affect cardiovascular contrast physiology. In the Fontan circulation larger doses of contrast (Up to $800 \mathrm{mg}$ iodine/kg patient weight) and delayed imaging appropriate to the cardiac output can result in a diagnostic single phase acquisition. Renal function needs to be considered as a large contrast dose can lead to contrast-induced nephropathy; which is a $25 \%$ increase from baseline creatinine within 48-72 h or increase in serum creatinine concentration greater than $0.5 \mathrm{mg} / \mathrm{dL}(44 \mathrm{micromol} / \mathrm{L})$ in the absence of another clear cause of kidney injury [63].

\section{Specific indications}

\section{Vascular stent patency}

In contrast to $\mathrm{CMR}, \mathrm{CT}$ angiography allows the assessment of vascular anatomy patency maintained with stents containing high ferro-magnetic material. However, the artefact produced by platinum stents is more pronounced in CT, making assessment of the stents difficult [64]. Thus it is important to note the types of stents that have been used in these patients.

\section{Coronary artery imaging}

The high spatial resolution of cardiac CT can accurately evaluate the coronary artery anatomy in patients from neonates to adults [65]. It has been suggested that CT coronary angiogram is more accurate than MR angiography in detecting coronary disease [66]. However, in contrast to a standard CT coronary protocol, there may be a need to modify the acquisition timing as the contrast transit time through the pulmonary circulation may be slower than expected.

\section{Fontan circuit patency}

It is difficult to perform a single study, which optimises coronary imaging and subsequent Fontan circuit patency, due to different dose requirements for each. However, a single contrast phase, biphasic acquisition protocol (usually ECG gated heart then helical volume) may be adequate. A standard dose of $400 \mathrm{mg} / \mathrm{kg}$ administered for twenty seconds should ensure optimal opacification of the coronary arteries if acquisition is timed by either bolus tracking from the descending aorta or performing a test bolus. A second delayed acquisition allows for contrast recirculation and opacification in the central veins to image the inferior Fontan circuit (Figs. 8 and 9). Coronary/aortic opacification is optimised at the expense of increased radiation dose from two acquisitions. The drawback of this technique is the standard contrast volume for optimal coronary imaging may be too low for adequately opacifying the inferior Fontan, for which a high contrast volume is often required.

\section{Thrombus}

There are two options to identify inferior Fontan circuit thrombus, both requiring a high volume of contrast to ensure adequate inferior Fontan circuit opacification when contrast recirculates. A biphasic contrast, single (helical or ECG gated volume) acquisition ensures simultaneous opacification of superior and inferior Fontan circuits. An initial large volume of contrast with rapid flow rate followed immediately by a smaller volume delivered continuously at a lower flow rate such that the total contrast injection time is around sixty seconds. This prevents the problems of contrast mixing/streaming artefact and the pseudothrombus phenomenon when the recirculated contrast enters the inferior Fontan circuit (Fig. 10). Overall cardiac chamber enhancement is usually good and homogenous but may not be as dense as an arterial timed, more rapidly injected early phase scan. 


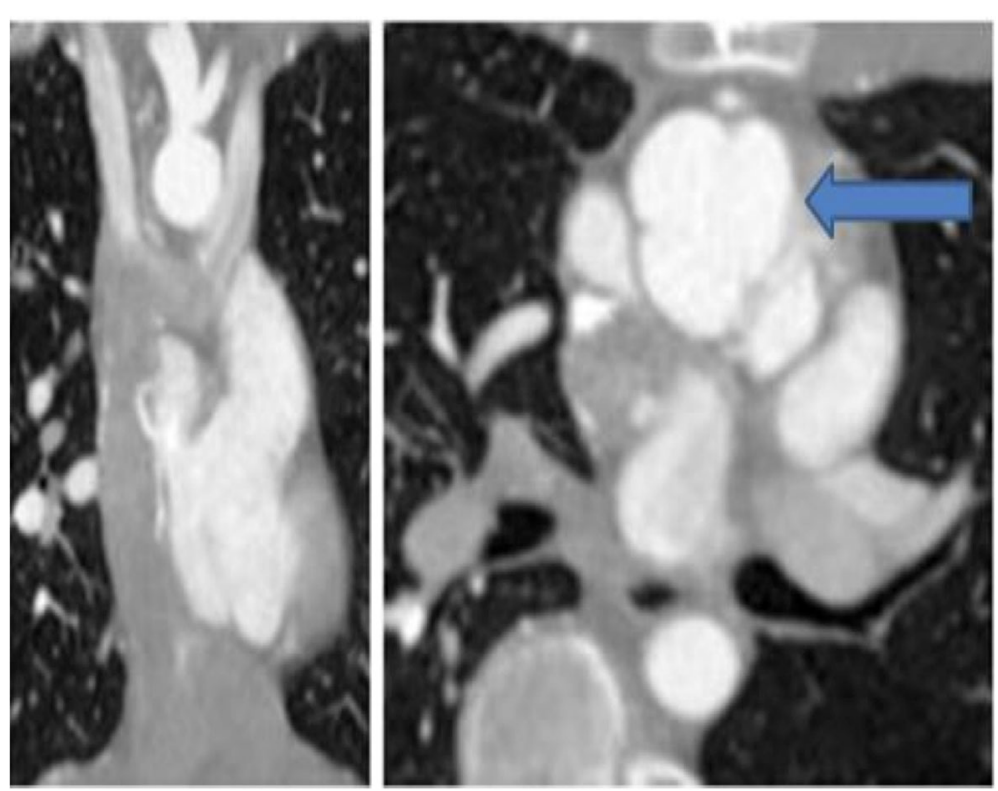

Fig. 8 Extra-cardiac Fontan. Single contrast phase, bi-phasic acquisition protocol. Early arterial phase. The arrow indicates a well opacified aortic root

Aortopulmonary collaterals and pulmonary arteriovenous malformations can also be adequately imaged.

Another option is to administer a high volume of contrast, for example $800 \mathrm{mg} / \mathrm{kg}$ over $40 \mathrm{~s}$, and scan at 80 $100 \mathrm{~s}$. Whilst the first pass contrast from the injection would have cleared the superior Fontan circuit, recirculating contrast from the head and neck should opacify the superior connection adequately.

\section{Ventricular function}

In the presence of contraindications for a CMR, ECGgated cardiac CT ventricular volumetrics obtained at 2 phases of the cardiac cycle (end-diastole and end- systole) showed good agreement with measurements obtained using CMR [67] and can (with appropriate consideration of and attention to radiation dose) be used in place of CMR to evaluate cardiac function as well as the more usual vascular anatomy.

\section{Patent Fontan fenestration}

Imaging of a patent fenestration is difficult on crosssectional imaging and is seen better with direct angiography on cardiac catheter. On cross-sectional imaging this is reliant on differences in contrast opacification which may result in pooling of contrast, increases in density or jets of opacified blood from one chamber to

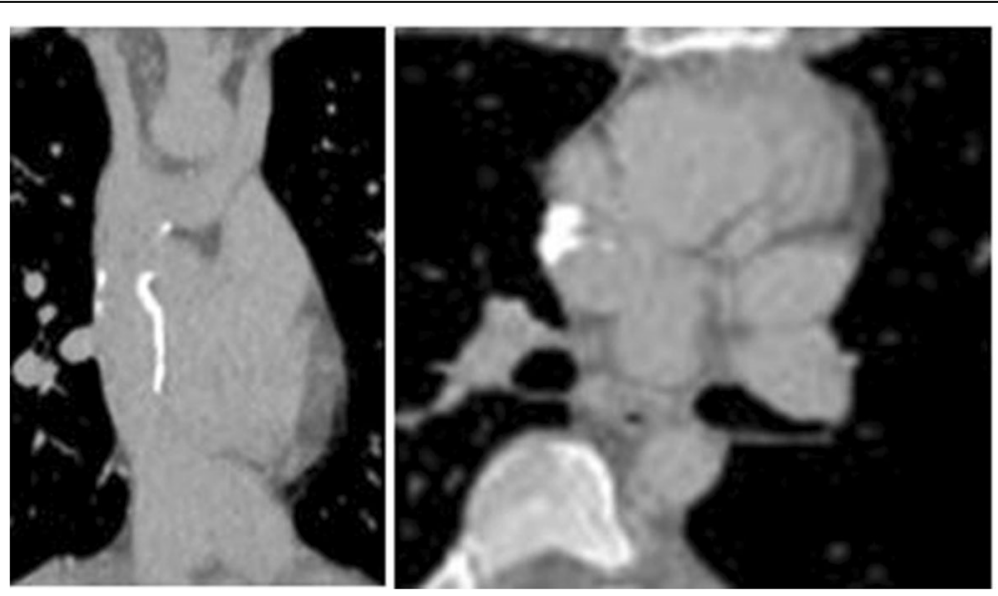

Fig. 9 Extra-cardiac Fontan circuit, same patient. Single contrast phase, bi-phasic acquisition. Late venous phase. Contrast has re-circulated to opacify the inferior Fontan connection 


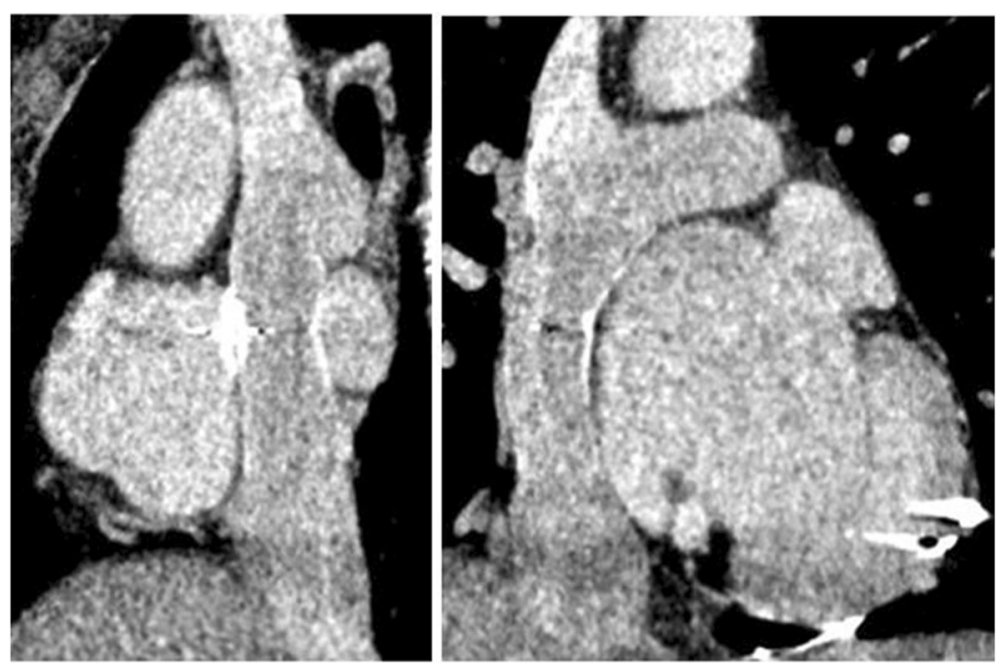

Fig. 10 Extra-cardiac Fontan circuit. Biphasic contrast phases, single acquisition. Both superior and inferior Fontan circuits opacified

another; 'negative' jets of unopacified blood may also be seen if flow is reversed into the opacified side. Early imaging is likely to be non-diagnostic with an arm injection. Late imaging may aid anatomical defect detection and may also show change in contrast opacification if a long injection is administered.

\section{Myocardial fibrosis}

Contrast opacification kinetics using cardiac CT can be appreciated [68]; similar to gadolinium and myocardial scar imaging; where CMR cannot be used. However, this technique is not fully validated and would require an additional acquisition thus increasing the radiation burden further.

\section{Common pitfalls}

There are several pitfalls to imaging Fontan circuits with $\mathrm{CT}$.

1) The failing ventricle, more notably the systemic RV, can impair the flow of intravenous contrast through the heart resulting in a rather 'washed out' study. In reality, there is lack of wash-in. Severe valve regurgitation may result in contrast pooling.

2) Dysrhythmias almost invariably result in some cardiac motion blurring which, whilst may affect coronary imaging, may not be a problem for gross anatomical imaging. The faster scanner with better temporal resolution can aid with this. A heart rate of less than $65 \mathrm{bpm}$ in sinus rhythm produces optimal image quality. Fontan patients may have a relative tachycardia and have a relatively fixed stroke volume rendering the use of beta blockade less predictable than in normal subjects. If it is not known to be safe to give beta blockade and the heart rate is elevated greater than about 75 beats per minute, cardiac gating may be best achieved in end systole (rather than mid diastole). The dysrhythmic patient invariably results in a higher radiation dose study than the patient in a regular rhythm due to the inability of the scanner to predict the $\mathrm{R}$ wave and enable good prospective ECG-gating, or the inability to effectively dose-modulate the study to deliver a lower dose during the systolic phases.

3) Inadequate field of view can be an issue. It is important to consider proximal SVC and inferior Fontan connections, which may extend beyond the 'normal' cardiac field of view. Additionally, patients with Fontan circuits may have additional congenital cardiovascular pathology such as superior- or infradiaphragmatic anomalous pulmonary venous connections or aortic coarctation. Whilst these may be identified incidental to the primary pathology, a detailed cardiac history is vital to the imager, to ensure the field of view includes relevant anatomy.

\section{Conclusion}

Cross-sectional diagnostic imaging modalities such as CMR and Cardiac CT are vital in the management of patients with Fontan operation. These provide excellent understanding of the wide anatomical variance and physiological status that can help guide future medical therapy, catheter-based or surgical interventions. Newer imaging techniques will continue to evolve and may assist in the long-term prognostication of this interest group of patients.

A patient-tailored imaging strategy is often required and an appreciation by the referring clinician and cardiac imager of the relative merits and pitfalls of each modality important. 


\section{Abbreviations}

CHD: Congenital heart disease; CMR: Cardiac MRl; CT: Computed tomography; ECV: Extra-cellular volume fraction; GFR: Glomerular filtration rate; ICD: Implantable cardioverter-defibrillator; IVC: Inferior vena cava; LPA: Left pulmonary artery; MR: Magnetic resonance; MRA: MR angiography; MRI: Magnetic resonance imaging; NSF: Nephrogenic systemic fibrosis; PAVM: Pulmonary arteriovenous malformations; PPM: Permanent pacemaker; RPA: Right pulmonary artery; SSFP: Steady state free precession; SVC: Superior vena cava

\section{Acknowledgements}

Not applicable.

\section{Funding}

No source of funding.

\section{Availability of data and materials}

Data sharing not applicable to this article as no datasets were generated or analysed during the current study.

\section{Authors' contributions}

MY and WL drafted the manuscript. MH and NM reviewed the manuscript and helped modify the manuscript. All authors read and approved the final manuscript.

\section{Ethics approval and consent to participate}

Not applicable.

\section{Consent for publication}

Not applicable.

\section{Competing interests}

The authors declare that they have no competing interests.

\section{Publisher's Note}

Springer Nature remains neutral with regard to jurisdictional claims in published maps and institutional affiliations.

\section{Author details}

${ }^{1}$ Department of Paediatric Cardiology, Bristol Royal Hospital for Children, University Hospitals Bristol NHS Foundation Trust, Upper Maudlin St, Bristol BS2 8HW, UK. ²Radiology Department, Bristol Royal Infirmary, Bristol, UK. ${ }^{3}$ Cardiac MRI Unit, Bristol Heart Institute, Bristol, UK

Received: 4 January 2016 Accepted: 24 August 2017

Published online: 15 September 2017

\section{References}

1. Fontan F, Baudet E. Surgical repair of tricuspid atresia. Thorax. 1971:26:240-8.

2. de Leval MR, Deanfield JE. Four decades of Fontan palliation. Nat Rev Cardiol. 2010;7:520-7.

3. de Leval MR, Kilner P, Gewillig M, Bull C. Total cavopulmonary connection: a logical alternative to atriopulmonary connection for complex Fontan operations. Experimental studies and early clinical experience. J Thorac Cardiovasc Surg. 1998;96:682-95.

4. Marcelletti C, Corno A, Giannico S, Marino B. Inferior vena cava -pulmonary artery extracardiac conduit. A new form of right heart bypass. J Thorac Cardiovasc Surg. 1990;100:228-32.

5. Kawashima Y, Kitamura S, Matsuda H, Shimazaki Y, Nakano S, Hirose H. Total cavopulmonary shunt operation in complex cardiac anomalies. A new operation. J Thorac Cardiovasc Surg. 1984;87:74-81.

6. Setyapranata S, Brizard CP, Konstantinov IE, lyengar A, Cheung M, d'Udekem Y. Should we always plan a Fontan completion after a Kawashima procedure? Eur J Cardiothorac Surg. 2011;40:1011-5.

7. Prakash A, Powell AJ, Geva T. Multimodality noninvasive imaging for assessment of congenital heart disease. Circ Cardiovasc Imaging. 2010;3:112-25.

8. Lewis G, Thorne S, Clift P, Holloway B. Cross-sectional imaging of the Fontan circuit in adult congenital heart disease. Clin Radiol. 2015;70:667-75.

9. Simonetti OP, Finn JP, White RD, Laub G, Henry DA. "Black blood" T2-weighted inversion recovery MR imaging of the heart. Radiology. 1996;199:49-57.
10. Stehling MK, Holzknecht NG, Laub G, Bohm D, von Smekal A, Reiser M. Single shot T1- and T2-weighted magnetic resonance imaging of the heart with black blood: preliminary experience. MAGMA. 1996;4:231-40.

11. Carr JC, Simonetti O, Bundy J, Li D, Pereles S, Finn JP. Cine MR angiography of the heart with segmented true fast imaging with steady-state precession. Radiology. 2001;219(3):828-34.

12. Higgins $C B$, Holt W, Pfugfelder $P$, Sechtem U. Functional evaluation of the heart with magnetic resonance imaging. Magn Reson Med. 1998:6:121-39.

13. Su JT, Chung T, Muthupillai R, Pignatelli RH, Kung GC, Diaz LK, Vick GW, Kovalchim JP. Usefulness of real-time navigator magnetic resonance imaging for evaluating coronary artery origins in pediatric patients. Am J Cardiol. 2005;95(5):679-82.

14. Sorensen TS, Korperich H, Greil G, Eichhorn J, Barth P, Meyer H, Pederson EM, Beerbaum P. Operator independent isotropic three-dimensional magnetic resonance imaging for morphology in congenital heart disease: a validation study. Circulation. 2004;110:163-9.

15. Conte S, Gewillig M, Eyskens B, Dumoulin M, Daenen W. Management of late complications after classic Fontan procedure by conversion to total cavopulmonary connection. Cardiovasc Surg. 1999:7:651-5.

16. Khambadkone S. The Fontan pathway: what's down the road? Ann Pediatr Cardiol. 2008;1:83-92.

17. Brown DW, Powell AJ, Geva T. Imaging complex congenital heart disease functional single ventricle, the Glenn circulation and Fontan circulation: a multimodality approach. Prog Pediatr Cardiol. 2010;28:45-8.

18. Hashemi S, Parks WJ, Slesnick TC. 3D inversion recovery gradient echo respiratory navigator imaging using Gadofosveset Trisodium in a Fontan Ygraft patient. Int J Cardiovasc Imaging. 2014;30:993-4.

19. Longmore DB, Underwood SR, Hounsfield GN, Bland C, Poole-Wilson PA, Denison D, Klipstein RH, Firmin DN, Watanabe M, Fox K, Rees RSO, Mcneilly AM, Burman ED. Dimensional accuracy in magnetic resonance in studies of the heart. Lancet. 1985;325(8442):1360-2.

20. Semelka RC, Tomei E, Wagner S, Mayo J, Caputo G, O'Sullivan M, Parmley WW, Chatterjee K, Wolfe C, Higgins CB. Interstudy reproducibility of dimension and functional measurements between cine magnetic resonance studies in the morphologically abnormal left ventricle. Am Heart J. 1990;119(6):1367-73.

21. Margossian R, Schwartz ML, Prakash A, Wruck L, Colan SD, Atz AM, Bradley T, Fogel MA, Hurwitz LM, Marcus E, Powell AJ, Printz BF, Puchalski MD, Rychik J, Shirali G, Williams R, Yoo SJ, Geva T. Comparison of Echocardiographic and cardiac magnetic resonance imaging measurements of functional single ventricular volumes, mass, and ejection fraction (from the pediatric heart network Fontan cross-sectional study). Am J Cardiol. 2009;104(3):419-28.

22. Fratz S, Chung T, Greil GF, Samyn MM, Taylor AM, Buechel ERV, Yoo SJ, Powell AJ. Guideline and protocols for cardiovascular magnetic resonance in children and adults with congenital heart disease: SCMR expert consensus group on congenital heart disease. J Cardiovasc Magn Reson. 2013;15:51.

23. Lyen S, Mathias H, McAlindon E, Trickey A, Rodrigues J, Bucciarelli-Ducci C, Hamilton M, Manghat N. Optimising the imaging plane for right ventricular magnetic resonance volume analysis in adult patients referred for assessment of right ventricular structure and function. J Med Imaging Radiat Oncol. 2015;59(4):421-30.

24. Fogel MA, Weinberg PM, Chin AJ, Fellows KE, Hoffman EA. Late ventricular geometry and performance changes of functional single ventricle throughout stage Fontan reconstruction assessed by magnetic resonance imaging. J Am Coll Cardiol. 1996;28:212-21.

25. Rathod RH, Prakash A, Kim YY, Germanakis IE, Powell AJ, Gauvreau K, Geva T. Cardiac magnetic resonance parameters predict transplantation-free survival in patients with Fontan circulation. Circ Cardiovasc Imaging. 2014;7:502-9.

26. Prakash A, Travison TG, Fogel MA, Hurwitz LM, Powell AJ. Printz, Puchalski MD, Girish SS, Yoo SJ, Geva T. Relation of size of secondary ventricles to exercise performance in children after Fontan operation. Am J Cardiol. 2010; 106(11):1652-6.

27. Sabry AF, Reller MD, Silberbach GM, Rice MJ, Sahn DJ. Comparison of four Doppler echocardiographic methods for calculating pulmonary to systemic shunt flow ratios in patients with ventricular septal defects. Am J Cardiol. 1995;75(8):611-4.

28. Powell AJ, Maier SE, Chung T, Geva T. Phase-velocity cine magnetic resonance imaging measurement of pulsatile blood flow in children and young adults: in vivo and in vitro validation. Pediatr Cardiol. 2000:21(2):104-10.

29. Grosse-Wortmann L, Al-Otay A, Yoo SJ. Aortopulmonary collaterals after bidirectional Cavopulmonary connection or Fontan completion: quantification with MRI. Circ Cardiovasc Imaging. 2009;2:219-25. 
30. Whitehead KK, Gillespie MJ, Harris MA, Fogel MA, Rome JJ. Noninvasive quantification of systemic-to-pulmonary Colalteral flow. A major source of inefficiency in patients with superior Cavopulmonary connections. Circ Cardiovasc Imaging. 2009;2:405-11.

31. Markl M, Frydrychowicz A, Kozerke S, Hope M, Wieben O. 4D flow MRI. J Magn Reson Imaging. 2012;36(5):1015-36.

32. Haggerty CM, Restrepo M, Tang E, de Zelicourt D, Sundareswaran KS, Mirabella BJ, Whitehead KK, Fogel MA, Yoganathan AP. Fontan hemodynamics from 100 patient-specific cardiac magnetic resonance studies: a computational fluid dynamics analysis. J Thorac Cardiovasc Surg. 2014;148:1481-9.

33. Khiabani RH, Whitehead KK, Han D, Restrepo M, Tang E, Bethel J, Paridon SM, Fogel MA, Yoganathan AP. Exercise capacity in single-ventricle patients after Fontan correlates with haemodynamic energy loss in TCPC. Heart. 2015:101:139-43.

34. Haggerty CM, Kanter KR, Restrepo M, de Zelicourt DA, Parks WJ, Rossignac J, Fogel MA, Yoganathan AP. Simulating hemodynamics of the Fontan Y-graft based on patient specific in vivo connections. J Thorac Cardiovasc Surg. 2013:145:663-70.

35. Rathod RH, Prakash A, Powell AJ, Geva T. Myocardial fibrosis identified by cardiac Magentic resonance late gadolinium enhancement is associated with adverse ventricular mechanics and ventricular tachycardia late after Fontan operation. J Am Coll Cardiol. 2010;55:1721-8.

36. Cheitlin MD, Robinowitz M, McAllister H, Hoffman JI, Bharati S, Lev M. The distribution of fibrosis in the left ventricle in congenital aortic stenosis and coarctation of the aorta. Circulation. 1980;62:823-30.

37. Chowdhury UK, Sathia S, Ray R, Singh R, Pradeep KK, Venugopal P. Histopathology of the right ventricular outflow tract and its relationship to clinical outcomes and arrhythmias in patients with tetralogy of Fallot. J Thorac Cardiovasc Surg. 2006;132:270-7.

38. Riesenkampff E, Messroghli DR, Redington AN, Grosse-Wortmann L. Myocardial T1 mapping in pediatric and congenital heart disease. Circ Cardiovasc Imaging. 2015;8:e0002504.

39. Moon JC, Dr M, Kellman P, Piechnik SK, Robson MD, Ugander M, Gatehouse PD, Arai AE, Friedrich MG, Neubauer S, Schulz-Menger J, Schelbert EB. Society for Cardiovascular Magnetic Resonance Imaging; cardiovascular magnetic resonance working Group of the European Society of cardiology. Myocardial T1 mapping and extracellular volume quantification: a Society for Cardiovascular Magnetic Resonance (SCMR) and CMR working Group of the European Society of cardiology consensus statement. J Cardiovasc Magn Reson. 2013;15:92.

40. Rosenthal DN, Friedman AH, Kopf GS KCS, Rosenfeld L, Hellenbrand WE. Thromboembolic complications after operation operations. Circulation. 1995:92:287-93

41. Coon PD, Rychik J, Novello RT, Ro PS, Gaynor JW, Spray TL. Thrombus formation after Fontan operation. Ann Thorac Surg. 2001;71:1990-4.

42. van Nieuwenhuizen RC, Peters M, Lubbers LJ, Trip MD, Tijssen JG, Mulder BJ. Abnormalities in liver function and coagulation profile following the Fontan procedure. Heart. 1999:82(1):40-6.

43. Casolo G, Rega L, Gensini GF. Detection of right atrial and pulmonary artery thrombosis after the Fontan procedure by magnetic resonance imaging. Heart. 2004;90:825.

44. Weinsaft JW, Kim HW, Shah DJ, Klem I, Crowley AL, Brosnan R, James OG, Patel MR, Heitner J, Parker M, Velazquez EJ, Steenbergen C, Judd RM, Kim RJ. Detection of left ventricular thrombus by delayed enhancement cardiovascular magnetic resonance. J Am Coll Cardiol. 2008:52:148-57.

45. Tsang W, Johansson B, Salehian O, Holm J, Webb G, Gatzoulis MA, Therrien J. Intracardiac thrombus in adults with the Fontan circulation. Cardiol Young. 2007;17(6):646-51.

46. Blaimer M, Breuer F, Mueller M, Heidemann RM, Griswold MA, Jakob PM. SMASH, SENSE, PILS, GRAPPA: how to choose the optimal method. Top Magn Reson Imaging. 2004;15(4):223-36.

47. Johansson B, Babu-Narayan SV, Kilner PJ. The effects of breath-holding on pulmonary regurgitation measured by cardiovascular magnetic resonance velocity mapping. J Cardiovasc Magn Reson. 2009;11:1.

48. Mascarenhas NB, Muthupillai R, Cheong B, Pereyra M, Flamm SD. Fast $3 \mathrm{D}$ cine steady-state free precession imaging with sensitivity encoding for assessment of left ventricular function in a single breath-hold. AJR. 2006;187:1235-9.

49. Krishnamurthy R, Pednekar A, Atweh LA, Vogelius E, Chu ZD, Zhang W, Maskatia S, Masand P, Morris SA, Krishnamurthy R, Muthupillai R. Clinical validation of free breathing respiratory triggered retrospectively cardiac gated cine balanced steady-state free precession cardiovascular magnetic resonance in sedated children. J Cardiovasc Magn Reson. 2015;17:1.

50. Barkhausen J, Goyen M, Ruhm SG, Eggebrecht H, Debatin JF, Ladd ME. Assessment of ventricular function with single breath-hold real-time steadystate free precession cine MR imaging. Am J Roentgenol. 2002;178:731-5.

51. Garg R, Powell AJ, Sena L, Marshall AC, Geva T. Effects of metallic implants on magnetic resonance imaging evaluation of Fontan palliation. Am J Cardiol. 2005;95:688-91.

52. Sasaki T, Hansford R, Menekhem MZ, Kolandaivelu A, Bluemke DA, Berger RD, Calkins $\mathrm{H}$, Halperin HR, Nazarian S. Quantitative assessment of artifacts on cardiac magnetic resonance imaging of patients with pacemakers and implantable Cardioverter-defibrillators. Circ Cardiovasc Imaging. 2011;4:662-70.

53. Brignole $M$, Auricchio A, Baron-Esquivias G, Bordachar P, Boriani G, Breithardt O, Cleland J, Deharo JC, Delgado V, Elliott PM, Gorenek B, Israel CW, Leclercq C, Linde C, Mont L, Padeletti L, Sutton R, Vardas PE. ESC guidelines on cardiac pacing and cardiac resynchronization therapy: the task force on cardiac pacing and resynchronization therapy of the European Society of Cardiology (ESC). Developed in collaboration with European heart rhythm association (EHRA). Europace. 2013;15(8):1070-118.

54. Kalin R, Stanton MS. Current clinical issues for MRI scanning of pacemaker and defibrillator patients. Pacing Clin Electrophysiol. 2005;28:326-8.

55. Langman DA, Goldberg IB, Finn JP, Ennis DB. Pacemaker lead tip heating in abandoned and pacemaker attached leads in 1.5 Tesla MRI. J Cardiovasc Magn Reson. 2011;33(2):426-31.

56. Ahmed FZ, Morris GM, Allen S, Khattar R, Mamas M, Zaidi A. Not all pacemakers are created equal: MRI conditional pacemaker and lead tehnology. J Cardiovasc Electrophysiol. 2013;24(9):1059-65.

57. van der Graaf AWM, Bhagirath P, Gotte MJW. MRI and cardiac implantable electronic devices; current status and required safety conditions. Neth Heart J. 2014;22:269-76.

58. Cheong BYC, Muthupillai R. Nephrogenic systemic fibrosis. Tex Heart Inst J. 2010;37(5):508-15.

59. Shellock FG, Spinazzi A. MRI safety update 2008: part 1, MRI contrast agents and nephrogenic systemic fibrosis. Am J Roentgenol. 2008;191(4):1129-39.

60. Kanda T, Fukusato T, Matsuda M, Toyoda K, Oba H, Kotoku J, Haruyama T, Kitajima K, Furui S. Gadolinium-based contrast agent accumulates in the brain even subjects without severe renal dysfunction: evaluation of autopsy brain specimens with inductively coupled plasma mass spectroscopy. Radiology. 2015;276(1):228-32.

61. Errante Y, Cirimele V, Mallio CA, Di Lazarro V, Zobel BB, Quattrochi CC. Progressive increase of $\mathrm{T} 1$ signal intensity of the dentate nucleus on unenhanced magnetic resonance images is associated with cumulative doses of intravenously administered gadodiamide in patients with normal renal function, suggesting dechelation. Investig Radiol. 2014;49(10):685-90.

62. Walsh MA, Noga M, Rutledge J. Cumulative radiation exposure in pediatric patients with congenital heart disease. Pediatr Cardiol. 2015;36(2):289-94.

63. Tepel M, Aspelin P, Lamiere N. Contrast-induced nephropathy: a clinical and evidence-based approach. Circulation. 2006;113:1799-806.

64. Kohler M, Burg MC, Bunck AC, Heindel W, Seifarth H, Maintz D. Dual-source CT angiography of peripheral arterial stents: in vitro evaluation of 22 different stent type. Radiol Res Pract. 2011;2011:103873.

65. Goo HW, Seo DM, Yun TJ, Park JJ, Park IS, Ko JK, Kim YH. Coronary artery anomalies and clinically important anatomy in patients with congenital heart disease: multislice CT findings. Pediatr Radiol. 2009;39:265-73.

66. Schuetz GM, Zacharopoulou NM, Schlattmann P, Dewey M. Meta-analysis: non-invasive coronary angiography using computed tomography versus magnetic resonance imaging. Ann Intern Med. 2010;152(3):167-77.

67. Yamamuro M, Tadamura E, Kubo S, Toyoda H, Nishina T, Ohba M, Hosokawa R, Kimura T, Tamaki N, Komeda M, Kita T, Konishi J. Cardiac functional analysis with multi-detector row $\mathrm{CT}$ and segmental reconstruction algorithm: comparison with echocardiography, SPECT and MR imaging. Radiology. 2005;234:381-90.

68. Watabe H, Sato A, Nishina H, Hoshi T, Sugano A, Kakefuda Y, Takaiwa Y, Aihara $H$, Fumikura $Y$, Noguchi $Y$, Aonuma K. Enhancement patterns detected by multidetector computed tomography are associated with microvascular obstruction and left ventricular remodelling in patients with acute myocardial infarction. Eur Heart J. 2016;37:684-92.

69. Mondesert B, Marcotte F, Mongeon FP, Dore A, Mercier LA, Ibrahim R, Asgar A, Miro J, Poirier N, Khairy P. Fontan circulation: success or failure. Can J Cardiol. 2013;29(7):811-20. 